\title{
Konkurrenz und Integration in Reorganisationsprozessen
}

\author{
Zur Problematik ,schöpferischer Zerstörung“ innerhalb von Organisationen
}

\section{Von Hans J. Pongratz}

Zusammenfassung: Reorganisationsprozesse generieren innerbetriebliche Konkurrenzverhältnisse, die sich mit Schumpeter als „schöpferische Zerstörung nach innen“ interpretieren lassen. Veränderungsprojekte setzen die Strukturen, Prozesse und Strategien einer Organisation immer wieder neuen Bewährungsproben aus. Der verdeckte Wettstreit um die Neukonzeption von Teilen der Organisation öffnet diese für ständige Anpassungsanforderungen, ohne bewährte Handlungsmuster vorschnell preiszugeben. Diese theoretische Interpretation der Konkurrenzdynamik und ihrer Integrationsproblematik wird entwickelt auf der Grundlage von Befunden der empirischen Reorganisationsforschung (einschließlich einer Befragung aller privatwirtschaftlichen Großbetriebe in Deutschland zu Problemen tief greifender Veränderungsprozesse). Deren scheinbar widerspruchsvolle Ergebnisse werden als Ausdruck kompetitiver Kooperation gedeutet, die nicht nur in Reorganisationsprojekten, sondern in Projektarbeit generell zu beobachten ist. Die kompetitive Dynamik von Projektarbeit weckt unternehmerische Energien bei Managern und Mitarbeitern und gibt zugleich Anlass, diese durch aktive Koordinierungsleistungen auf gemeinsame Ziele auszurichten.

\section{Perspektivenwechsel in der Reorganisationsforschung}

Reorganisationsprozesse sind verwickelte Angelegenheiten. Manager und Mitarbeiter in Unternehmen verschiedenster Branchen, in Nonprofit-Organisationen oder in staatlichen Behörden haben in den letzten Jahren ähnliche Botschaften vernommen: Der Wandel von Strategien, die Veränderung von Organisationsstrukturen oder Arbeitsabläufen werden von der Organisationsleitung als neue Ära angekündigt und mit hochgesteckten Effizienzzielen verknüpft. Das Überleben der Organisation hänge vom Erfolg solcher Umwälzungen ab, bekommen sie in der Regel zu hören. Dafür müssten Opfer gebracht werden (vor allem im Zuge von Personalabbau und Leistungsverdichtung), doch im Gegenzug stünden ansehnliche Belohnungen in Aussicht (etwa in Form gesicherter Arbeitsplätze oder erweiterter Verantwortungsbereiche).

Was Manager und Mitarbeiter (damit sind in diesem Text immer Männer wie Frauen gemeint) dann oft erleben, ist ein geschäftiges Treiben in eigens geschaffenen Projektgruppen, das ihnen als Außenstehenden weitgehend undurchsichtig bleibt. Unter der Anleitung von Unternehmensberatungen werden die zu reorganisierenden Bereiche durchleuchtet und neu konzipiert. Da die Mehrheit der Betroffenen nicht so recht weiß, welche Veränderungen mit welchen Absichten im Projekt vorbereitet werden, bleibt sie auf Distanz zum Reorganisationsgeschehen. Dem Aktionismus von Beratern und Projektverantwortlichen gegenüber nimmt sie eine abwartende Beobachterposition ein: Die Alltagsarbeit müsse schließlich weiterhin erledigt werden, ist eine verbreitete Haltung, und wie das wirkungsvoll geschehe, das wüssten immer noch die Manager und Mitarbeiter vor Ort am besten.

Unterdessen wird das Projekt - innerhalb eines engen Zeitrahmens mit mehr oder weniger Verspätung - zum Abschluss gebracht mit der Verkündigung des erfolgreichen Vollzugs von Reorganisationsmaßnahmen. Was das faktisch bedeutet, ist zu diesem Zeitpunkt meist unklar, da die konzipierten Veränderungen nur bedingt mit den Erfahrungen und Interessen der betroffenen Manager und Mitarbeiter in Einklang zu bringen sind. Die Auseinandersetzung mit den Projektergebnissen bleibt nun ihnen überlassen: So werden manche Veränderungskonzepte in wesentlichen Teilen realisiert, andere stark modifiziert, und wieder andere 
verlieren sich im Alltagsgeschäft und sind spätestens beim nächsten Reorganisationsprojekt vergessen.

Den vorliegenden Forschungsbefunden zufolge (siehe Kap. 2) stellt diese Skizze ein realistisches Szenario eines typischen Reorganisationsprozesses dar. Organisationsmitglieder aller Ebenen berichten von Erfahrungen dieser Art - und von damit einhergehenden Verunsicherungen und Enttäuschungen. Das Auftreten solcher Probleme ist aus sozialwissenschaftlicher Perspektive nicht verwunderlich: Die Neugestaltung grundlegender Strukturen und Prozesse stellt einen gravierenden Eingriff in das weit verzweigte Gewebe routinisierter Abläufe und bewährter Kooperationsmuster dar. Unter der Annahme komplexer Organisationssysteme und dynamischer Umwelten ist nicht zu erwarten, dass Reorganisationskonzepte friktionslos implementiert werden können und die geplanten Resultate zeitigen (vgl. Collins 1998; Kieser / Hegele / Klimmer 1998; Dawson 2003). Die geschilderten Probleme zeugen von der vielfach unterschätzten Schwierigkeit der Aufgabe.

Überraschend sind weniger die Probleme an sich, sondern dass sie im Verlauf von nunmehr gut zwanzig Jahren Reorganisationsforschung immer wieder in ähnlicher Form und anhaltender Frequenz festzustellen sind. Das gilt für Veränderungen der unterschiedlichsten Organisationsbereiche und ungeachtet ständig wechselnder Managementmoden und Beratungskonzeptionen. Obwohl die Syndromatik früh beschrieben wurde und in der Managementliteratur (z.B. Kanter / Stein / Jigg 1992) wohl bekannt ist, scheinen kaum Konsequenzen daraus gezogen worden zu sein. Mehr noch: Trotz vielfach beklagter Erfolgsdefizite von Veränderungsmaßnahmen ist der Aufwand dafür kräftig gesteigert worden, wie die Umsatzzahlen der Beratungsbranche belegen (Salaman 2002; Faust 2005). Auch für die nähere Zukunft wird eine Zunahme von Reorganisation erwartet (Pongratz / Trinczek 2006: 112f) und durch die Unternehmensergebnisse legitimiert: Die wirtschaftliche Leistungsfähigkeit steigt und auch besonders ertragreiche Organisationen halten an weiteren Umstrukturierungen fest. ${ }^{1}$

Die Forschung zur Reorganisation ist ebenso vielfältig wie unübersichtlich ${ }^{2}-$ und konnte bisher nur wenig zur Vermeidung wiederkehrender Umsetzungsprobleme und zur Behebung anhaltender Erfolgsdefizite beitragen. Die meisten empirischen Studien konzentrieren sich als Fallstudien auf einzelne Veränderungsmaßnahmen oder Organisationen. Da sie fast ausschließlich mit qualitativen Erhebungs- und Auswertungsmethoden arbeiten, zeichnen sie zwar ein anschauliches Bild typischer Reorganisationsprobleme, sind jedoch kaum systematisch miteinander vergleichbar und wenig verallgemeinerungsfähig (siehe Kap. 2.1). Die wenigen Metaanalysen, die bislang verfügbar sind, beschränken sich selektiv auf spezifische

1 Die diversen Ursachen und Anlässe von Reorganisationsmaßnahmen werden in der folgenden Analyse nicht behandelt. Im weitesten Sinne treibt der Wettbewerbsdruck einer globalisierten kapitalistischen Ökonomie die Unternehmen zu immer neuen Umstrukturierungen (vgl. z.B. Kanter / Stein / Jigg 1992). Neben der Bewältigung interner Probleme sind auch externe Erwartungen an die ständige Anpassungsbereitschaft von Organisationen zu erfüllen, die in vielen Fällen zur Nachahmung von Reorganisationsmoden führen (vgl. Kieser 1996).

2 Sie ist vorwiegend interdisziplinär angelegt - mit Schwerpunkten in der betriebswirtschaftlichen Managementlehre, der Sozialpsychologie und der Soziologie. Die Ursprünge dieser Forschungsrichtung liegen in den sozialpsychologischen Untersuchungen des Verhaltens in Gruppen durch den Kreis um Kurt Lewin in den 1940er Jahren. Eine breitere Basis gewann sie in der Fundierung praxisnaher Ansätze zur Veränderung von Organisationsstrukturen in den 1960er und 1970er Jahren, zum Beispiel im Konzept der Organisationsentwicklung (French / Bell 1994). In den 1980er Jahren erlangten Berater und Managementanalytiker schnelle Berühmtheit (und den Status von Management-Gurus) mit wissenschaftlich durchaus relevanten, aber normativ ausgerichteten Veränderungsrezepten (u.a. Peters / Waterman 1983; Kanter / Stein / Jigg 1992; Kotter 1996). Die parallel sich entwickelnde empirische Forschung fand mit ihren weniger plakativen Erkenntnissen weit geringere Aufmerksamkeit. 
Forschungsbereiche oder regionale Schwerpunkte (siehe Damanpour 1991; Armenakis / Bedeian 1999; Sauer / Boes / Kratzer 2005; Caldwell 2005).

Die Vergleichbarkeit der Befunde leidet überdies unter der unzureichenden organisationstheoretischen Fundierung der gesamten Forschungsrichtung. Das herkömmliche organisationstheoretische Instrumentarium liefert zwar eine Reihe von Ansatzpunkten zur Analyse organisatorischen Wandels (vgl. als Übersicht Demers 2007), aber zur neuen Dimension des permanenten Wandels von Organisationen sind allenfalls erste Interpretationsansätze vorhanden. ${ }^{3}$ Die Frage, warum es trotz allen Erfahrungszuwachses nicht gelungen ist, die Reorganisationsproblematik zuverlässiger zu meistern, ist so bisher nicht zufriedenstellend beantwortet. Deshalb wird im Folgenden ein Wechsel der Perspektive vorgeschlagen: Wenn die geschilderten Schwierigkeiten den Unternehmenserfolg nicht verhindert und die Wandlungsdynamik nicht gebremst haben, dann sind sie selbst möglicherweise ein substanzieller Aspekt der Lösung. Sie zeugen nicht einfach (zumindest nicht vorrangig) von Lernunfähigkeit oder unzureichendem Veränderungsmanagement. Vielmehr sind sie Ausdruck und Begleiterscheinung eines neuen Organisationsprinzips, nämlich einer spezifischen Form der Konkurrenz innerhalb von Organisationen.

Dieser Perspektivenwechsel wird verknüpft mit Schumpeters Theorem von der ,schöpferischen Zerstörungsleistung“ des Unternehmers als Triebkraft der Erneuerung kapitalistischer Märkte (Kap. 3.1). In formaler Analogie zu Schumpeter (bei erheblichen inhaltlichen Differenzen) wird die Reorganisationsdynamik als organisationsinterne Konkurrenzsituation interpretiert, die produktive Energien weckt (vgl. auch Pongratz 2008). Da die Theorie Konkurrenzphänomene innerhalb von Organisationen bisher weitgehend ignoriert hat, muss das analytische Instrumentarium für ein solches Phänomen erst noch entwickelt werden. Vorläufige Überlegungen dazu führen zum Begriff der kompetitiven Kooperation (Kap. 3.2) und der Frage nach der Motivation kompetitiver Leistungen (Kap. 3.3). Abschließend wird die Problematik der organisatorischen Integration derartiger Konkurrenzbeziehungen erörtert (Kap. 4). Hat die Organisationstheorie den Wandel bisher als Übergangsphänomen des prinzipiell stabilen Organisationsgefüges gesehen, so postuliert dieser Perspektivenwechsel, dass permanente Reorganisation durch die Etablierung unregulierter, aber produktiver Konkurrenzbeziehungen zum Normalzustand von Organisationen werden kann. Dieser Blickwechsel ermöglicht zum einen eine stringente Deutung der disparaten empirischen Befunde der Reorganisationsforschung und liefert zum anderen Ansatzpunkte für die erforderliche Theorieentwicklung. Zunächst wird (in Kap. 2) mit der Präsentation maßgeblicher Forschungsbefunde die empirische Ausgangslage skizziert.

\section{Forschungsbefunde zu den unbewältigten Problemen der Reorganisation}

Nach einem Überblick über zentrale Befunde der internationalen empirischen Forschung zu Reorganisationsprozessen (Kap. 2.1) soll etwas ausführlicher auf die Ergebnisse einer eigenen Erhebung zu tief greifenden Veränderungsprozessen in deutschen Großbetrieben eingegangen werden (Kap. 2.2). Zwar greift auch diese Studie nur auf die subjektiven Einschätzungen betrieblicher Experten zu (und nicht auf objektive Unternehmensdaten), aber auf der Grundlage einer repräsentativen Unternehmensauswahl liefert sie ein - wenn auch sehr gro-

3 Caldwell (2005) spricht in seinem Überblick über konzeptionelle Zugänge der Reorganisationsforschung deshalb nicht von Theorien, sondern von ,competing disciplinary discourses on agency and change in organizations, classified into rationalist, contextualist, dispersalist and constructionist discourses“ (2005: 83). Engere Theoriebezüge finden sich in kritischen Analysen zu Reorganisationskonzepten, z.B. von Collins (1998). Im Rahmen der Critical Management Studies wird die kritische Gesellschaftstheorie (v.a. im Anschluss an Foucault und Habermas) zur Interpretation empirischer Reorganisationsanalysen genutzt (z.B. Knights / Willmott 2000; Clark / Fincham 2002). 
bes - Gesamtbild der Problematik. Zudem waren es die Resultate dieser Studie, die Anlass zu einer intensiveren Auseinandersetzung mit dem Verhältnis von Konkurrenz und Integration in Unternehmen gegeben haben.

\subsection{Ergebnisse der empirischen Reorganisationsforschung im Überblick}

Die Befunde der internationalen Forschung lassen sich in drei Themensträngen bündeln, die erstens die Effektivität von Interventionsstrategien, zweitens deren ungeplante soziale Dynamik und drittens die Reaktionen der betroffenen Organisationsmitglieder im Fokus haben.

(1) Zur Optimierung des Change-Managements wurde früh nach entscheidenden Gestaltungsoptionen für den Erfolg von Reorganisationsprojekten gefahndet. ${ }^{4}$ Im Mittelpunkt stehen häufig die Einflussmöglichkeiten einzelner Managementgruppen:

- Ein wesentlicher Faktor ist die Kommunikationsstrategie des Top-Managements (Pongratz / Trinczek 2006: 118). Als erfolgreich werden Unternehmensleiter beschrieben, die eine positive Vision des Veränderungsanliegens vermitteln (z.B. in Form einer „New Vision Speech“, Armenakis / Harris / Mossholder 1993: 697) und zugleich einen realistischen Blick für praktische Umsetzungsprobleme behalten (Pettigrew 1987: 666). Als Machtpromotoren (zur Unterscheidung von den Fachpromotoren siehe Witte / Hauschildt / Grün 1988) stehen sie für die Dringlichkeit und Entschiedenheit des Veränderungsanspruchs ein. Die Wirksamkeit solcher Botschaften gilt als höher, wenn sie in direktem persönlichem Kontakt vermittelt werden und die Möglichkeit zum Gespräch einschließen (Foehrenbach / Goldfarb 1990; Bernecker / Reiß 2002).

- Die vielfältigen Rollenanforderungen und Qualifikationsvoraussetzungen so genannter Change Agents gerieten frühzeitig in den Fokus der Reorganisationsforschung (siehe Wooten / White 1989) und führten zu diversen Typisierungsversuchen (Tichy / Hornstein 1976; Ottaway 1983). Es zeigt sich, dass Change Agents aufgrund der Beschränkungen ihrer formalen Machtbefugnisse auf laterale Netzwerkbildung und informelle Einflusstaktiken (,,influence rather than command“, Hartley / Benington / Binns 1997: 71) angewiesen sind.

- Im Vergleich zur Erfolgsrelevanz der Kommunikation von Unternehmensleitung und Projektteam erweist sich die Bedeutung des Projektmanagements und der Organisationsberatung als unerwartet gering (vgl. Greif / Runde / Seeberg 2004: 291ff). Ausreichende Ressourcenausstattung und effiziente Projektorganisation erscheinen im Urteil von Managern als notwendige Voraussetzung, aber keineswegs als hinreichende Bedingung für den Veränderungserfolg (Pongratz / Trinczek 2006: $117 \mathrm{f}$ ). Da Evaluationen von Reorganisationsprozessen selten sind, ist auch die Rolle der (internen und externen) Berater noch wenig erforscht; Fallstudien belegen ihre Verstrickung in mikropolitische Auseinandersetzungen (Iding 2000).

(2) Aus der schwierigen Suche nach Erfolgsfaktoren erwuchs die Einsicht, dass organisatorischer Wandel nur in Grenzen planmäßig steuerbar und durch zentrale Instanzen beherrschbar ist. Die Aufmerksamkeit richtete sich deshalb verstärkt auf die komplexe soziale Eigendynamik von Reorganisationsprozessen:

4 Methodisch ist der Nachweis von Erfolgsfaktoren für ein komplexes soziales Geschehen schwierig. Auch für die Reorganisationsforschung gilt die Kritik, die generell an der Erfolgsfaktorenforschung in der Managementlehre geübt wird (siehe March / Sutton 1997; Nicolai / Kieser 2002): Sowohl die Messung des Veränderungserfolgs (als abhängiger Variable) als auch die Operationalisierung möglicher Erfolgsfaktoren (als unabhängigen Variablen) wirft beträchtliche Probleme auf (die etwa bei Greif / Runde / Seeberg 2004 erörtert werden). Die Erfolgsfaktoren, die immer wieder in privat finanzierten Studien ausgewiesen werden (siehe z.B. Capgemini 2005; IBM 2007), beruhen in der Regel auf deskriptiven Analysen mit ungeklärter Stichprobenbasis. 
- Im Mittelpunkt steht die Frage nach den Ursachen und Formen jenes Widerstands von Mitarbeitern und Managern gegen Reorganisationsprojekte, der in den meisten Fallstudien in mehr oder weniger ausgeprägter (und offener) Weise zutage tritt. ,Resistance to change“, von Lewin (1947) noch als gruppendynamischer Effekt begriffen, wurde oft als vorsätzliche Gegnerschaft gedeutet, die es zu überwinden gelte. Zunehmend werden die ambivalenten Motive vieler Betroffener und das konstruktive Potenzial ihrer veränderungskritischen Haltungen erkannt (als Überblick siehe Dent / Goldberg 1999 und Piderit 2000). Neben Verlustängsten bezüglich Arbeitsplatz und Karriere führen vor allem Verunsicherungen hinsichtlich Leistungsanforderungen und Kooperationsbeziehungen zu distanzierten Haltungen (Armenakis / Bedeian 1999: 304ff).

- Der Dissens zwischen engagierten Change Agents und zurückhaltenden Betroffenen gibt Anlass zu Aushandlungsprozessen und Konflikten, in denen ein ganzes Arsenal von Handlungsstrategien und Machtmitteln zum Einsatz kommen kann (Buchanan / Badham 1999). Für Change Agents wird machtpolitisches Taktieren zur systematischen Rollenanforderung (ebd.: 624). Die Vielzahl der beteiligten Akteure mit unterschiedlichen Interessenlagen erzeugt unübersichtliche mikropolitische Konstellationen, deren Konfliktlinien und Koalitionsbildungen von den in der Organisation üblichen Kontroversen oft abweichen; Arrangements und taktische Machtbalancen sind eher von kurzer Dauer (Ortmann et al. 1990: 395ff).

(3) Im Gefolge derartiger Analysen reiften realistischere Einschätzungen des Reorganisationsgeschehens heran, wonach viele Veränderungsprojekte nicht nur ihre Ziele verfehlen, sondern auf die Betroffenen geradezu lähmend wirken (zur Psychodynamik der Erwartungsstrukturen im Organisationswandel siehe Becke 2008). Die Erforschung der Reaktionen von Managern und Mitarbeitern liefert ein ernüchterndes Resultat:

- Der durch Reorganisationsmaßnahmen verursachte Stress hängt mit der Infragestellung bewährter Routinen zusammen (Armenakis / Bedeian 1999: 306 f); bisher erfolgreiche Praktiken und Kompetenzen werden als entwertet empfunden. Die Verunsicherung ergreift auch viele Führungskräfte, die sich unklaren und widersprüchlichen Rollenanforderungen ausgesetzt sehen und den Veränderungsprozess als Zumutung erleben (Kotthoff 1996; Faust / Jauch / Notz 2000).

- Reorganisationen lösen starke Emotionen aus, die von enthusiastischer Zustimmung bis zu bitterer Enttäuschung reichen (Finstad 1998: 731). Positive Gefühle stehen eher im Zusammenhang mit dem alltäglichen Arbeitsumfeld, während negative Gefühle primär Enttäuschung vom oberen Management ausdrücken (Kiefer / Müller / Eicken 2001; Kiefer 2002). Manager der mittleren Ebenen (ebenso wie Gewerkschaftsvertreter im Betrieb) können durch „emotional balancing“ zur Bewältigung dieses Spannungsverhältnisses beitragen, indem sie mit einem realistischen positiven Commitment offen bleiben für Bedenken und Ängste der Mitarbeiter (Huy 2002).

- So unterschiedlich wie die Emotionen fallen auch die Handlungsmuster der Betroffenen aus (Armenakis / Bedeian 1999: 309 f), wobei eher von spontanen situativen Arrangements als von gezielten Bewältigungsstrategien auszugehen ist (Orlikowski 1996). Ein typisches Muster ist: „temporary adoption, but ultimate rejection of the new behaviors necessary to achieve lasting success" (Armenakis / Bedeian 1999: 310). Im Extremfall von „excessive change“, vor allem bei sich in widersprüchlicher Weise überlagernden Veränderungsanforderungen, werden Reaktionen von passivem Gehorsam bis hin zu Sabotage beobachtet (Stensaker et al. 2002: 303ff) - sowie besonders häufig BOHICA (,,bend over, here it comes again“) als Lernerfahrung aus vergangenen Projekten, die da lautet: in Deckung gehen und abwarten ,until this wind of change had blown over" (ebd.: 304). 
Im Zusammenhang gesehen vermitteln diese Befunde jenes eingangs skizzierte Bild einer verwickelten und aufreibenden sozialen Konstellation: anspruchsvolle Veränderungsvisionen der Geschäftsführung (die aber nicht geschlossen vertreten werden), motivierte Change Agents (denen Überlastung durch eine Vielzahl von Aufgaben droht), verunsicherte Führungskräfte und abwartende Mitarbeiter. Machtauseinandersetzungen und emotionale Belastungen zeugen von einer intensiven sozialen Dynamik und von erheblichen Problemen ihrer Bewältigung. Die Gleichzeitigkeit von Zentralisierungs- und Dezentralisierungsbestrebungen (vgl. Kühl 2002; Pongratz 2008) erzeugt zusätzliche Widersprüche: Ergebniserwartungen werden zentral verfügt und organisationsstrategisch verfolgt, operative Gestaltungsformen werden dezentral entwickelt und mikropolitisch verhandelt. Auf welche Weise die auseinanderstrebenden Bewegungen wieder organisatorisch integriert werden, lässt die bisherige Forschung noch kaum erkennen (vgl. Kap. 4). ${ }^{5}$ Doch können diese Probleme keineswegs mehr als vorübergehende Begleiterscheinungen begrenzter Transformationen gelten (wie es zunächst den Anschein haben mochte), sondern sie werden als dauerhaftes Organisationsphänomen erkennbar, das permanente Anpassungsfähigkeit erzwingt (Kratzer 2003).

\subsection{Reorganisationsprobleme in deutschen Großbetrieben}

Mit einer Befragung deutscher Großunternehmen (Vollerhebung aller privatwirtschaftlichen Betriebe mit mehr als 1000 Mitarbeitern) im Jahr 2006 wollten wir nicht die genannten Forschungsdefizite beheben, sondern ein (notwendig pauschal bleibendes) Gesamtbild der Reorganisationsproblematik zeichnen. ${ }^{6}$ Ziel der Studie war ein systematischer Überblick über Erfolgsquoten und zentrale Problemfaktoren tief greifender Veränderungsprozesse: strategische Neuausrichtung, grundlegende Restrukturierung, Kulturwandel sowie Mergers \& Acquisitions. Bezüglich der Messung des Reorganisationserfolgs ebenso wie hinsichtlich der Berücksichtigung der Diversität von Veränderungsprozessen steht sie vor denselben Herausforderungen wie die Erfolgsfaktorenforschung (siehe Fußnote 4). Das Kernproblem ist der Mangel an objektiven Unternehmensdaten zu Verlauf und Ergebnis von Umstrukturierungsmaßnahmen. Auch die hier durchgeführte schriftliche Expertenbefragung muss sich auf subjektive Urteile von Projektverantwortlichen stützen, von denen zwar kompetente Einschät-

5 Für die Forschungslage trifft weiterhin die Einschätzung Pettigrews zu, ,that, with a few limited and noteworthy exceptions [...], much research on organization change is ahistorical, aprocessual, and acontextual in character" (1987: 655). Die Berücksichtigung historischer Konstellationen, dynamischer Prozessverläufe und konkreter Kontextbedingungen würde indessen die Komplexität des Erklärungsanspruchs erheblich steigern - und nicht nur hohe Anforderungen an die Datengewinnung stellen, sondern wesentlich erweiterte Theoriegrundlagen voraussetzen.

6 Die Studie wurde von der Unternehmensberatung C4 Consulting GmbH, Düsseldorf, in Auftrag gegeben und als Forschungsbericht veröffentlicht (Houben et al. 2007). Beteiligt waren Rainer Trinczek und Christa Herrmann von der TU München sowie Hans Pongratz, Christoph Biester und Maren Täger von der LMU München. An die Geschäftsführer und Vorstände aller privatwirtschaftlichen Großbetriebe in Deutschland (insgesamt 1017 Betriebe entsprechend der Betriebsstatistik der Bundesagentur für Arbeit) wurde die Bitte gerichtet, den Fragebogen an Führungskräfte weiterzureichen, die tief greifende Veränderungsprozesse geleitet hatten. Da jedes Unternehmen drei Fragebögen erhielt, kamen aus einigen Betrieben mehrere Antworten. In der Zeit von Mai bis Juli 2006 gingen insgesamt 201 gültige Fragebögen aus 167 Unternehmen ein, was einer Rücklaufquote (bezogen auf die Zahl der Betriebe) von $16 \%$ entspricht. Gemessen an den Kriterien Betriebsgröße und Branche weist das Untersuchungssample einen hohen Grad an Repräsentativität auf; leicht überrepräsentiert sind sehr große Unternehmen sowie die Finanzbranche. 
zungen erwartbar sind, die dennoch von persönlichen Eindrücken und partikularen Veränderungsmotiven bestimmt bleiben. ${ }^{7}$

Abb. 1: Analytisches Modell zur Erklärung von Veränderungserfolg (unter Angabe der in der Regressionsanalyse untersuchten Einflussfaktoren)

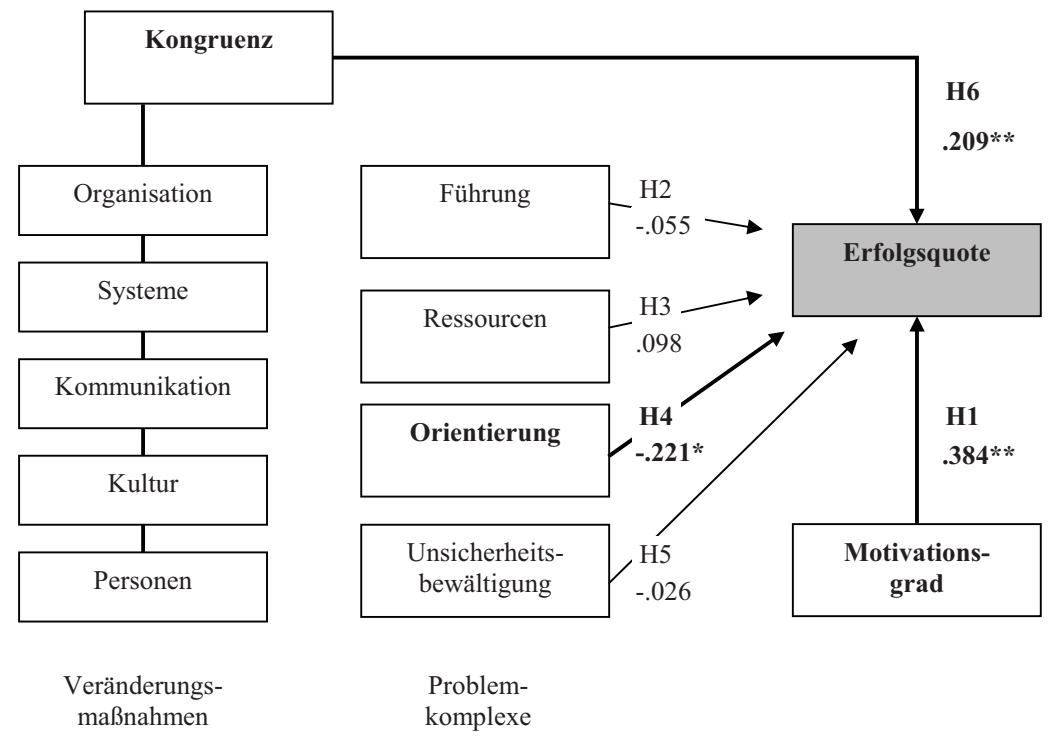

Auf der Grundlage der qualitativen Reorganisationsforschung (siehe Kap. 2.1) - aber wegen der geschilderten Theoriedefizite ohne explizite theoretische Begründung - wurde vorab ein exploratives analytisches Modell zum Zusammenhang zentraler Problemfaktoren mit dem Veränderungserfolg entwickelt (Abb. 1). Diesem Modell liegen drei Basisannahmen zugrunde:

- Annahme A: Der Reorganisationserfolg hängt primär von der Veränderungsmotivation ab (H1); zahlreichen Fallstudienbefunden zufolge werden Veränderungsprozesse durch mangelnde Beteiligung der Betroffenen massiv beeinträchtigt.

- Annahme B: Als Einflussfaktoren sowohl auf die Motivation als auch auf den Veränderungserfolg werden in der Reorganisationsforschung vier zentrale Problemkomplexe identifiziert: Führungsprobleme (H2) und mangelnde Ressourcenausstattung (H3), Defizite der Orientierung (H4) und der Unsicherheitsbewältigung (H5) reduzieren den Veränderungserfolg.

- Annahme C: Art und Umfang von Veränderungsmaßnahmen beeinflussen die genannten Zusammenhänge: Generell steht eine Reduzierung von Maßnahmen und eine entsprechend geringe Kongruenz (Ganzheitlichkeit im Sinne von Breite und Häufigkeit der Maßnahmen) dem Reorganisationserfolg im Wege (H6).

7 Auch für erfahrene Projektmanager ist es schwierig, im Großbetrieb einen Überblick über sämtliche Reorganisationsprozesse zu gewinnen und ein zuverlässiges Urteil zum Gesamtgeschehen abzugeben. Aufgrund der eigenen Projektverantwortung sind eine Überschätzung des Erfolgs und die Unterschätzung von Problemen zu erwarten. Diese Verzerrungstendenz wird bestätigt durch Kontrollfragen zu dem zuletzt geleiteten Veränderungsprojekt. Die Befragten zählen größtenteils zum Top-Management (25\% Geschäftsführung, $30 \%$ Bereichsleitung) und verfügen über eine langjährige Betriebszugehörigkeit (von durchschnittlich 14 Jahren) bei einem Durchschnittsalter von 45 Jahren. 
Die Auswertung liefert zunächst eine Reihe aufschlussreicher deskriptiver Befunde (zur Operationalisierung der Variablen und zu weiteren Resultaten siehe Houben et al. 2007). Die Schätzungen zur Erfolgsquote von Reorganisation bestätigen die verbreitete Problemdiagnose: Nur 30 \% der Veränderungsprozesse werden im Durchschnitt als ,voll erfolgreich“, 41 \% als ,überwiegend erfolgreich“ gewertet. Demgegenüber werden zwar lediglich $10 \%$ der Prozesse als ,gescheitert“ befunden, aber gemeinsam mit $20 \%$,wenig erfolgreichen“ Prozessen gilt ein knappes Drittel der Veränderungsprojekte als Problemfall. ${ }^{8}$ Noch etwas skeptischer fällt das Expertenurteil zur Veränderungsmotivation der Belegschaft aus: Knapp die Hälfte der Mitarbeiter gilt als entweder „,nicht motiviert“ (16\%) oder „wenig motiviert“ (29\%). „Eher motivierte“ (37\%) und ,voll motivierte“ (19\%) Mitarbeiter werden zwar in der Mehrheit gesehen, aber für eine breite Mitwirkung an Umstrukturierungsmaßnahmen sprechen auch diese Werte nicht.

Abb. 2: Problempunkte nach der Rangfolge der subjektiv geschätzten Erfolgsrelevanz (Angaben in Prozent)

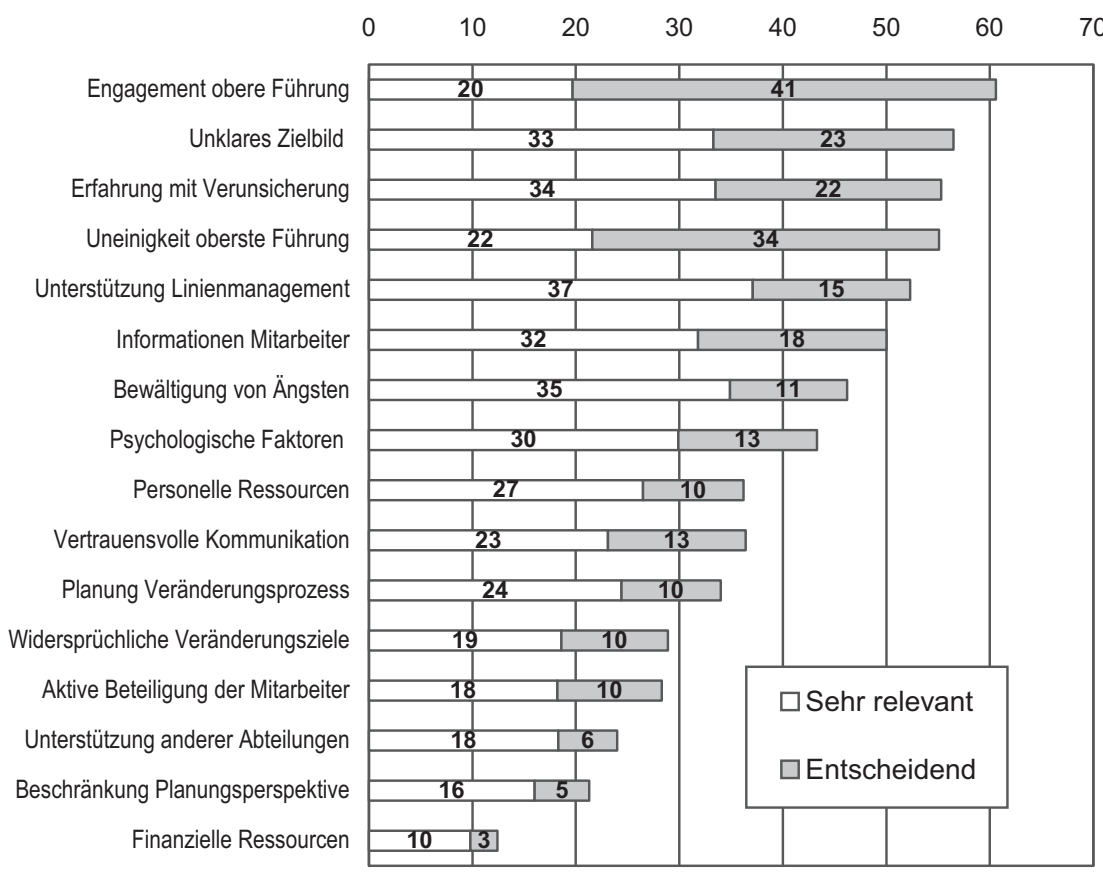

Als den wichtigsten Problemkomplex identifizieren die Experten den Faktor „Führung“. In einer Liste von 16 Problempunkten (Abb. 2) rangiert das konsequente Auftreten der Führungsspitze an vorderster Stelle: In ,entscheidender“ Weise behindern demnach den Veränderungserfolg ,unzureichendes Engagement der oberen Führungsebenen“ (41\%) und „Unei-

8 Die Frage zur Schätzung der Erfolgsquote lautet: „Wenn Sie an alle tief greifenden Veränderungsprozesse denken, die Sie in Ihrem Unternehmen kennen gelernt haben, zu welcher Gesamteinschätzung kommen Sie? Wie viel Prozent dieser Veränderungsprozesse schätzen Sie ein als (von insgesamt $100 \%$ ): voll erfolgreich ... \%, überwiegend erfolgreich ...\%, wenig erfolgreich ...\%, gescheitert ... \%?" Der Motivationsgrad wird analog dazu durch die Schätzung der Prozentanteile der Belegschaft hinsichtlich von vier Motivationsstufen gemessen. 
nigkeit auf den obersten Führungsebenen (sprechen nicht mit einer Stimme)“ (34 \%). Für die addierten Werte „sehr relevant" und „entscheidend“ (Abb. 2) liegen die Items des Faktors „Führung“ auf den Rängen 1, 4, 5 und 6. Den zweitwichtigsten Problemkomplex aus Sicht der Experten bildet die „Unsicherheitsbewältigung“ (Rang 3, 7, 8, 10 und 14), gefolgt von den Faktoren „Orientierung“ (Rang 2, 11, 12 und 15) und „Ressourcen“ (Rang 9, 16). Neben inkonsequenter Führung wird also vor allem der unzulängliche Umgang mit den psychischen Belastungen der Reorganisation für Veränderungsprobleme verantwortlich gemacht. Diese Bewertungen der deutschen Führungskräfte stimmen in bemerkenswertem Maße mit den Erkenntnissen der Reorganisationsforschung überein.

Die Annahmen des analytischen Modells zum Zusammenhang von Veränderungsmaßnahmen und Problemkomplexen mit Erfolgsquote und Motivationsgrad werden durch die Regressionsanalyse nur teilweise gestützt (siehe Abb. 1). Die Faktoren „Führung“ (H2), „Ressourcen“ (H3) und „Unsicherheitsbewältigung“ (H5) weisen nur schwache Zusammenhänge mit der Erfolgsquote auf. Nur der Faktor „Orientierung“ (H4) zeigt in der multivariaten Analyse einen signifikanten Einfluss auf die Erfolgsquote: Seine vier Merkmale „Klarheit der Zielbilder“", „Klarheit der Planungsperspektive“, „Widerspruchsfreiheit der Veränderungsziele" und „Beschränkung der Planungsperspektive“" kommen also relativ unabhängig vom Motivationsgrad zur Wirkung. Das sind alles Merkmale, die einen Veränderungsprozess als überlegtes, planvolles Vorgehen kenntlich machen und rational nachvollziehbar erscheinen lassen. Im Kontrast zur emotionalen Dimension von Motivation und Unsicherheitsbewältigung verweist der Orientierungs-Faktor auf die kognitive Dimension der Stimmigkeit von Richtung und Ziel der Veränderung, welche dem Vorhaben Sinn verleiht.

Neben dem Faktor „Orientierung“ weist die Regressionsanalyse den Motivationsgrad und die Kongruenz der Veränderungsmaßnahmen als unabhängige Einflussfaktoren auf die Erfolgsquote aus - und bestätigt damit die Hypothesen H1 und H6 (siehe Abb. 1). Erwartungsgemäß hat der Motivationsgrad den stärksten Einfluss auf die Erfolgsquote: Je höher die Experten den Anteil der motivierten Mitarbeiter einschätzen, umso größer fallen auch die angegebenen Anteile an erfolgreichen Veränderungsprozessen aus (H1). Die Kongruenz der Veränderungsmaßnahmen stellt neben dem Orientierungs-Faktor ein zweites bislang unterschätztes Wirkungsmoment dar. Mit Kongruenz wird die Ganzheitlichkeit der Veränderung in den Blick genommen: Wie umfassend und konsequent sind Veränderungsmaßnahmen angelegt?9 Beschränken sie sich auf die „harten“ Komponenten „Organisation“ und „Systeme“ oder schließen sie die „weichen“ Komponenten „Kommunikation“, „Kultur“ und „Personen" mit ein? Der Anteil erfolgreicher Prozesse wird von den befragten Experten höher angesetzt, wenn ,harte“ und ,weiche“ Komponenten zu einer ausgeprägten Kongruenz beitragen (H6).

Zusammenfassend betrachtet erweisen sich die drei Faktoren Motivation, Orientierung und Kongruenz als relativ unabhängig voneinander wirksame Einflüsse auf die (von betrieblichen Experten geschätzte) Erfolgsquote tief greifender Reorganisationsprozesse in deutschen Großbetrieben. In welcher Weise Orientierung und Kongruenz genau wirksam werden, ist mit dieser schriftlichen Befragung nicht zu klären und bedarf neuer Aufmerksamkeit in der Forschung. Alle drei Faktoren lassen sich indes verstehen als Hinweise auf die Relevanz von Integrationsleistungen in sich reorganisierenden Betrieben. Denn im Reorganisationsprozess geraten bewährte Handlungsmuster und Veränderungsansprüche in Widerspruch zueinander und divergierende Innovationsvorschläge stellen das gemeinsame Organisationsverständnis in Frage. Der Kongruenz-Faktor verweist auf die Anforderung, Veränderungs-

9 Gemessen wird die Kongruenz mit einem Index, der die Häufigkeit aller 13 abgefragten Veränderungsmaßnahmen erfasst. Auf die Bedeutung der Kongruenz von Veränderungsvorhaben, Umfeldbedingungen und Prozessmerkmalen für den Reorganisationserfolg hat Damanpour (1991) hingewiesen. 
maßnahmen wirkungsvoll aufeinander abzustimmen. Orientierung macht Verlauf und Richtung der Reorganisation nachvollziehbar und damit als sinnvoll erfahrbar. Und Motivation trägt zur Akzeptanz von Veränderungen bei - als Voraussetzung, um an deren Umsetzung konstruktiv mitzuwirken.

\section{Reorganisation als innerbetriebliche Konkurrenz}

Die geschilderte soziale Dynamik von Reorganisationsprozessen (Kap. 2.1) und der Beitrag von Integrationsleistungen zum Reorganisationserfolg (Kap. 2.2) lassen sich in einen sinnvollen Zusammenhang miteinander bringen, wenn sie als Ausdruck innerbetrieblicher Konkurrenzverhältnisse interpretiert werden. Dieses Argument soll in drei Schritten entwickelt und vertieft werden: Die Analogie zum Theorem ,schöpferischer Zerstörung“ betont den notwendigen Perspektivenwechsel (Kap. 3.1), der den Modus kompetitiver Kooperation (Kap. 3.2) und dessen Zusammenhang mit projektförmiger Arbeitsorganisation (Kap. 3.3) in den Blick rückt.

\subsection{Schöpferische Zerstörung nach innen}

In der Reorganisationsdynamik ist (beabsichtigt oder unbeabsichtigt) ein Konkurrenzverhältnis angelegt: Strategien, Strukturen und Prozesse werden unter dem Veränderungspostulat ständigen Bewährungsproben ausgesetzt, in denen sie entweder bestehen oder sich ändern müssen. Veränderungsprojekte schaffen Konkurrenzsituationen zwischen bestehenden Organisationselementen und deren Neukonzeption: Die Verfechter beider Seiten sind gehalten, ihre Ressourcen zu mobilisieren, um entweder erprobte Routinen zu behaupten oder Neuerungen durchzusetzen. ${ }^{10}$ Dieser Konkurrenzmechanismus bedeutet nicht kontinuierlichen Wandel (im Sinne etwa von Schreyögg / Noss 2000), sondern regelmäßige Konfrontation mit Veränderungsanforderungen bei prinzipiell offenem Ausgang. Die Funktion dieses Prinzips ist die Öffnung der Organisation für permanente Anpassungsoptionen an sich ändernde Umweltbedingungen (Märkte, Technologien, Managementmodelle, Arbeitskräfte usw.), ohne bewährte Handlungsmuster bedingungslos preiszugeben. Es erhöht die Flexibilität bei gleichzeitiger Beschränkung des Risikos vorschnellen und übermäßigen Wandels. In diesem Sinne können auch Reorganisationen, die nichts verändern, dann als wirkungsvoll gelten, wenn sie ein bestehendes Handlungsmuster als weiterhin geeigneten Lösungsansatz ausweisen. Der Projekterfolg hängt nicht von der Umsetzung eines Plans ab, sondern von der Aussagekraft des Wettstreits zwischen Erneuerungs- und Bewahrungsbestrebungen. ${ }^{11}$

Das der Reorganisation inhärente Konkurrenzprinzip lässt sich - in Analogie zu Schumpeters berühmtem Theorem - als „schöpferische Zerstörung nach innen“ verstehen. Schumpeter sieht im innovativen Unternehmer den Agenten jener schöpferischen Zerstörung, welche die kapitalistische Wirtschaft immer wieder von Neuem revolutioniert und damit ihre Entwicklung vorantreibt. Das „Erkennen und Durchsetzen neuer Möglichkeiten“ als „Wesen der Unternehmerfunktion“ (Schumpeter 1929: 483) richtet sich vorwiegend nach außen auf das Marktumfeld des Unternehmens, nämlich auf die Erschließung neuer Produkte, Absatzmärkte und Bezugsquellen (ebd.). Auf organisatorische Veränderungen in Form neuer Produktionsmethoden und überbetrieblicher Kooperationsformen (z.B. Vertrustung) weist

10 Zur Vereinfachung der Argumentation werden im Folgenden antagonistische Positionen unterstellt, obwohl realiter von einem Kontinuum von Haltungen zwischen Erneuerung und Bewahrung (mit vielen Zwischenpositionen) auszugehen ist.

11 Verschiedene Analysen bestätigen die Gleichzeitigkeit von Wandel und Stabilität in Reorganisationsprozessen: „Empirically and theoretically, change and continuity need one another“ (Pettigrew 1987: 649; vgl. auch Leana / Barry 2000 oder Huy / Mintzberg 2003). Hier wird sie interpretiert als Konkurrenzbeziehung zwischen Akteuren mit unterschiedlichen Interessen. 
Schumpeter hin, ohne diesen Aspekt weiter zu vertiefen. In seinem Spätwerk geht er (im Anschluss an Weber) von stabilisierten Organisationsstrukturen und mechanisierten Abläufen aus: Im bürokratisierten Betrieb habe sich der Unternehmer selbst überflüssig gemacht und seine Innovationsfunktion an spezialisierte Expertengruppen abgegeben (Schumpeter 1950: 213ff).

Im Widerspruch zur Bürokratisierungsannahme macht die Reorganisationsdynamik die Prozesse und Strukturen der Organisation selbst zum Gegenstand schöpferischer Zerstörung. Der unternehmerische Impuls richtet sich nach innen auf die Umwälzung des organisatorischen Kooperationsgefüges. Sein Erfolg entscheidet sich nicht im Marktmodus (wie im Außenverhältnis), sondern in der Konkurrenz zwischen Erneuerern und Bewahrern (im Innenverhältnis). Dabei wirkt der Anreiz zur kreativen Lösungssuche nicht nur für die Neuerer, sondern auch für deren Opponenten. Denn beide Seiten geraten unter den Druck, nach innovativen Kombinationen alter und neuer Elemente zu suchen, um ihre Grundposition durchzusetzen. Dass alles beim Alten bleibt, ist ebenso die Ausnahme wie eine vollständige und planmäßige Neugestaltung: Das Innovationspotenzial liegt in der Synthese von Alt und Neu - in jener erfinderischen Neukombination der Produktionsfaktoren, welche Schumpeter als die eigentlich unternehmerische Leistung erachtet. Das Theorem „schöpferischer Zerstörung“ trägt zum Verständnis von Reorganisationsprozessen bei, indem es die produktiven Potenziale von zunächst chaotisch und destruktiv erscheinenden Konkurrenzbeziehungen aufzeigt.

\subsection{Merkmale kompetitiver Kooperation}

Noch stellt die Konkurrenz zwischen Organisationsmitgliedern ein wenig systematisch behandeltes Gebiet der Organisationsforschung dar. Zwar ist der Kampf um Führungspositionen, mit denen in hierarchischen Organisationen der Aufstieg in verantwortungsvolle und lukrative Stellen (und der individuelle Karriereerfolg) verbunden sind, ein klassisches Thema. Allerdings hat sich das Forschungsinteresse mehr auf Ursachen und Verlaufsmuster von Karrieren als auf die Rivalität der Kollegen im Ausleseprozess gerichtet (vgl. Adamson / Doherty / Viney 1998; Sullivan 1999). Stärkere Aufmerksamkeit wird den Kollegenbeziehungen in interaktionistischen Studien zur Auseinandersetzung um die Gestaltung der Kooperationsstrukturen zwischen verschiedenen Berufsgruppen in professionalisierten Organisationen gewidmet (z.B. Strauss et al. 1963; Stelling / Bucher 1972). Anlass zu Konflikten geben demnach vor allem unterschiedliche berufsfachliche Kulturen und Statusansprüche; die Ergebnisse der Aushandlungsprozesse lassen sich mit Strauss (1978) als ,,negotiated order“ charakterisieren (siehe auch Fine 1984; Hall 1987). Zumeist aber wird die Konkurrenz um Ressourcen (Budgets, Verantwortungsbereiche) und Erträge (Lohn, Anerkennung) als Führungsproblem im Verhältnis von Management und Mitarbeitern und nicht als laterale Konkurrenz behandelt.

In Reorganisationsprozessen weist die Konkurrenz um Ressourcen und Erträge systematisch über klassische Fragen der Personalführung hinaus. Der besondere Modus des verdeckten Wettstreits um die Gestaltung des organisatorischen Wandels lässt sich als kompetitive Kooperation kennzeichnen. Damit wird betont, dass die Konkurrenz (a) nicht zwischen beliebigen Akteuren, sondern im Wesentlichen zwischen Organisationsmitgliedern (und deren Verbündeten) stattfindet und (b) den organisatorischen Rahmen nicht nur voraussetzt, sondern konstitutiv an seiner Erhaltung und Entwicklung mitwirkt. Kompetitive Kooperation weist (c) zudem umfassenden Charakter auf und erfasst potenziell alle Organisationsmitglieder - unabhängig von ihren Interessenpositionen.

Drei weitere Merkmale sind essenziell: (d) das experimentelle Prinzip, (e) die unbestimmten Entscheidungsbedingungen und (f) die Endlosigkeit des Wettstreits. Das experimentelle Prinzip kompetitiver Kooperation folgt der Logik von Versuch und Irrtum und erfordert eine 
Vielzahl von Lösungsversuchen: Teile der betrieblichen Handlungsarena werden frei gegeben für die Sondierung effizienter betrieblicher Gestaltungsansätze. Dabei bleiben die Entscheidungskriterien großenteils unbestimmt; sie können im Prozessverlauf variieren und sowohl Aspekte von Effizienz als auch von Legitimation und Macht beinhalten. Entscheidungen über „,erfolgreiche Lösungen“ beenden deshalb das Konkurrenzverhältnis nicht, sondern markieren lediglich Zwischenetappen in einem fortdauernden Wettstreit.

Aus organisationstheoretischer Sicht fallen neben den machtanalytischen Bezügen die Analogien derart verdeckt-experimenteller Konkurrenzbeziehungen mit dem Mülleimer-Modell des Entscheidungsverhaltens in mehrdeutigen Situationen vom Typus ,organisierter Anarchie" ins Auge: etwa bezüglich inkonsistenter Ziele, wechselnder Teilnehmer oder unklarer Entscheidungsregeln (Cohen / March / Olsen 1972; March / Olsen 1976). Doch steht hier (im Unterschied zum Mülleimer-Modell) nicht die Entscheidungsfindung im Mittelpunkt, sondern die Generierung innovativer Gestaltungsansätze aus Initiativen der Organisationsbasis. „Schöpferische Zerstörung nach innen“ mobilisiert die Kreativitätsressourcen der Belegschaft für die organisatorische Entwicklung relativ unabhängig von zentralen Entscheidungsverfahren. Hier kommt die Gleichzeitigkeit von Zentralisierungs- und Dezentralisierungstendenzen (siehe Kap. 2.1) zur Wirkung: Dezentrale Handlungsfelder werden unregulierter Konkurrenz ausgesetzt, um den Pool an Lösungsansätzen für zentrale Entscheidungen zu erweitern. Das Votum der Organisationsleitung leistet dann zwar eine formale Integration der Wettbewerbsresultate, beendigt aber nicht die durch die rivalisierenden Aktivitäten ausgelösten Turbulenzen.

Denn auch nach einer formalen Entscheidung bleibt die Dynamik kompetitiver Kooperation spürbar: Machtkämpfe wirken nach, Bündnisse und Gegnerschaften bestehen fort, Intransparenz von Entscheidungskriterien wird als Ungerechtigkeit bewertet, vergebliche Anstrengungen münden in Enttäuschung, nicht anerkannte kreative Leistungen werden trotzig weiterverfolgt. Dieses Unruhepotenzial droht destruktiv zu werden, wenn es nicht mehr in den Wettstreit innovativer Gestaltung eingebunden ist. Dieser Gefahr begegnet die Strategie permanenter Reorganisation, indem sie stets von neuem Umstrukturierungsprojekte zur nutzbringenden Verwertung der einmal geweckten kreativ-zerstörerischen Kräfte schafft. Empirische Forschungen (siehe v.a. Stensaker et al. 2002) lassen allerdings darauf schließen, dass auch diese Form der Einbindung auf längere Frist die Energien aufzehren und die Innovationsressourcen erschöpfen kann. Permanente Reorganisation ist kein Perpetuum mobile, das seine Antriebskraft auf Dauer aus sich selbst heraus erneuert.

\subsection{Projektförmige Arbeit als Organisationsmodus interner Konkurrenz}

Damit stellt sich die Frage, welche Handlungsmotive die skizzierte Konkurrenzdynamik in Gang halten. Schumpeter konzipiert schöpferische Zerstörung als Prozess, der durch die Tatkraft unternehmerischer Akteure vorangetrieben wird (Swedberg 1994). Als deren Motive zur innovativen Ressourcenkombination gibt er an: „der Traum und der Wille, ein privates Reich zu gründen“, „Siegerwille“ und „Freude am Gestalten“ (Schumpeter 1964: 138). Aus welchen Quellen speist sich die Innovationsenergie von Reorganisation (insbesondere wenn man die geschilderten Motivations- und Orientierungsprobleme in Rechnung stellt)? Ein ,,privates Reich“ lässt sich mit der Beteiligung an Veränderungsprozessen kaum schaffen: Gestalterische Initiativen führen innerhalb der Organisation nicht zur eigenständigen Unternehmung (wie unter Marktbedingungen), sondern bleiben auf die Bewilligung von Handlungsfreiräumen angewiesen. Auch für „Siegerwillen“ gibt es wohl ergiebigere Betätigungsfelder (innerhalb der Organisation beispielsweise den Aufstieg in der Hierarchie), selbst wenn die Konkurrenzsituation gelegentlich Wettbewerbscharakter annimmt. Als Motiv für innovatorisches Unternehmertum innerhalb von Organisationen ist in erster Linie die „Freude am Ge- 
stalten“ in Betracht zu ziehen. Denn durch Dezentralisierungsmaßnahmen werden organisatorische Handlungsbeschränkungen abgebaut und eigenständige Initiativen ermutigt.

In den empirischen Reorganisationsstudien ist eine hohe intrinsische Motivation von Change Agents vielfach belegt, aber bisher kaum systematisch untersucht. Eingehendere Analysen der Motivation zur gestalterischen Initiative in Organisationen stammen aus Studien zu selbstbestimmter Projektarbeit (vgl. Kalkowski / Mickler 2009). Projekte sind zeitlich befristete und ergebnisorientierte Kooperationszusammenhänge zur Bewältigung nicht-alltäglicher komplexer Aufgaben. In einer explorativen Studie zu Gruppen- und Projektarbeit in deutschen Unternehmen haben Pongratz und Voß (2003: 66ff) Leistungsorientierungen von Beschäftigten ermittelt, die gerade aus den schwer kalkulierbaren Anforderungen von Projekten emotionale Befriedigung (ihren „Spaß“ an der Arbeit) ziehen. Diese Haltung der „Leistungsoptimierung“ ist vom Anspruch der gemeinsamen Bewältigung einer ungewöhnlichen professionellen Herausforderung geleitet (vgl. auch das psychologische Konzept der Proaktivität: Crant 2000; Parker / Turner / Williams 2006). Trotz häufig bis an die Leistungsgrenzen reichender Belastungen sind diese Manager und Mitarbeiter willens, den eigenen Projektbeitrag in einem selbstgesteuerten Optimierungsprozess auf flexible Handlungserfordernisse abzustimmen. Dazu werden unternehmerische Handlungsstrategien eingesetzt, wie die Bereitschaft zum kalkulierten Risiko oder Improvisation zur kreativen Lösungsfindung.

Der Orientierungstypus „Leistungsoptimierung“ findet sich auch in anderen Arbeitsformen (vgl. Pongratz / Voß 2004), aber unter Bedingungen qualifizierter Projektarbeit scheinen die Voraussetzungen dafür besonders günstig zu sein (Pongratz / Voß 2003: 199ff). Denn Projekte bieten einerseits reizvolle und bei begrenzter Ressourcenausstattung recht anspruchsvolle Aufgaben und andererseits Handlungsspielräume für selbstbestimmtes Arbeiten und kreative Lösungssuche. In einer kultursoziologischen Analyse zeigen Boltanski und Chiapello (2006: 147ff), wie die ,projektbasierte Organisation“, die „,der Kreativität und der Innovation verpflichtet" (ebd.: 176) ist, in den 1990er Jahren zum vorherrschenden Modell der Managementliteratur geworden ist. Mit ihr verbinde sich ein unternehmerisches Verständnis von Aktivität im Sinne von ,etwas in Angriff nehmen, etwas unternehmen, sich verändern" (ebd.: 209 f). Projektarbeit schafft eher als die klassisch hierarchische Führungsposition Anreize, im Schumpeterschen Sinne unternehmerisch tätig zu werden.

Die Analogien von Ergebnissen zur innovativen Projektarbeit mit der vorgeschlagenen Interpretation der Innovationsdynamik von Reorganisation geben Anlass zur Annahme, dass in beiden Fällen ein ähnlicher Konkurrenzmechanismus wirksam ist - zumal auch Reorganisation in aller Regel in Projektform organisiert ist. Reorganisationsprojekte weisen sicherlich eine Reihe von Besonderheiten auf: Sie provozieren in besonderem Maße internen Widerstand, sofern sie eingespielte Verhaltensmuster außer Kraft zu setzen versuchen, und verursachen damit erhebliche Anpassungskosten (siehe Kap. 2). Der Spaß an der inhaltlichen Herausforderung verschleißt sich selbst bei erfahrenen Change Agents häufig im „,mikropolitischen Kleinkrieg“ mit dem Kollegenkreis. Marktbeziehungen und die Ansprüche externer Partner spielen dagegen meist nur eine geringe Rolle. Mit der Interpretation von Projektarbeit und Reorganisation als gleichartigen, aber unterschiedlich ausgeformten Modi kompetitiver Kooperation wird diese als grundlegendes Organisationsprinzip innovativer, dezentraler Leistungen bestimmt.

\section{Integration „von unten“ durch Koordination kompetitiver Leistungen}

Bleibt abschließend zu klären, warum die durch Projektarbeit entfesselten Kräfte (direkt oder indirekt) kompetitiver Optimierungsansprüche an die Arbeitsgestaltung nicht das Organisationsgefüge sprengen - und konsequenter Weise in Marktbeziehungen münden. Bei Schumpeter sorgt der Markt dafür, dass die „schöpferische Zerstörung“ der Unternehmer der 
wirtschaftlichen Entwicklung insgesamt zugute kommt: Die für die Marktteilnehmer vorteilhaften Angebote behaupten sich in interessengeleiteten Tauschakten und führen dazu, dass nicht konkurrenzfähige Angebote letztlich vom Markt genommen werden. Innovatives unternehmerisches Handeln innerhalb der Organisation erfordert eine marktäquivalente Koordination, die nur bedingt durch zentrale Steuerung erfolgen kann und dezentrale Adaptionshandlungen voraussetzt. Die Annahme ist deshalb, dass die miteinander konkurrierenden Organisationsmitglieder zugleich die Integration ihrer Leistungen anstreben - und dass die Projektform nicht nur zu kompetitiver Arbeit anspornt, sondern zugleich deren wirkungsvolle Koordination ermöglicht.

Wenn die Konkurrenzdynamik von Projektarbeit und Reorganisation ähnlich ist, können auch vergleichbare Integrationsmechanismen wirksam werden. Grundsätzlich gilt: Je ideenreicher und eigenwilliger Manager und Mitarbeiter ihre dezentralen Initiativen entwickeln, umso schwieriger sind sie in eine zentral gelenkte Unternehmensstrategie einzubinden. Die klassischen Integrationsformen liefern dafür keine hinreichende Erklärung (ohne dass ihre prinzipielle Wirksamkeit bestritten werden soll): Formale Steuerung per Hierarchie und Leistungsnormen kann ebenso wie der (weniger formale) Einfluss der Organisationskultur einem innovativ optimierenden Gestaltungsanspruch - je nach Situation - förderlich oder hinderlich sein. Und die Permanenz der Reorganisation generiert zwar beständige Optimierungsanreize, bedingt aber auch eine Menge frustrierender Erfahrungen. Die Spezifik kompetitiver Kooperation erfordert zusätzlich zu diesen Einbindungsformen einen spezifischen Integrationsmodus.

Durch kompetitive Kooperation verändert sich das Verhältnis von Individuum und Organisation: Kompetitive Leistungen sind durch den organisatorischen Rahmen angeregt und eingehegt, aber ihr kreatives Potenzial können sie nur über eigenständige Initiativen von Managern und Mitarbeitern entfalten. Die kompetitive Dynamik geht von den Individuen aus. Gleichzeitig lässt die empirisch belegte Haltung der Leistungsoptimierung auf aktive Integrationsleistungen ,von unten“ schließen: Manager und Mitarbeiter gehen nicht nur ihre unmittelbare Arbeitsaufgabe unternehmerisch an, sondern sorgen aus eigenem Willen und in schöpferischer Weise zugleich dafür, dass ihr individueller Arbeitsbeitrag eine effektive Eingliederung in den kollektiven Kooperationszusammenhang erfährt. Denn nur wenn diese Eingliederung gelingt, können sie ihr Selbstverständnis als selbstständig und professionell handelnde Akteure behaupten. Diese Interpretation lässt sich stützen durch die (leider noch spärlichen) empirischen Befunde zur Projektarbeit: ${ }^{12}$ Projekte entwickeln regelmäßig ein Eigenleben, das zwar nicht absichtlich gegen die Organisation gerichtet ist (zumal es letzten Endes deren Zielen dient), das sich aber von formalen Regelungen sukzessive löst. Die Einschränkung der Verfügungsrechte der Linienvorgesetzten über Projektmitarbeiter ist Ausdruck dieser Abkoppelung. Eben diese Verselbstständigung soll jene kreativen Potenziale wecken, welche die besondere Leistungsfähigkeit von Projekten ausmachen.

Reorganisationsprojekte sind mit der Zielsetzung der Änderung organisatorischer Routinen als Sonderfall von Projektarbeit zu betrachten. Sie schaffen die paradoxe Situation, dass der offizielle Auftrag die Aufhebung bestehender Normen verlangt und alle, die an ihnen festhalten, zu delegitimierten Oppositionellen macht. Übereinstimmung mit Organisationsnormen ist so zumindest während der Umstellungsphase schwer zu erreichen, weil bislang konformes Handeln im Verlauf des Veränderungsprozesses eine grundlegende Umwertung er-

12 Da die spezifischen Bedingungen von Projektarbeit empirisch wenig untersucht sind (siehe z.B. Becker-Beck / Fisch 2001; Bollinger 2001; Hodgson 2002; Latniak / Gerlmaier 2006; Hodgson / Cicmil 2006; Kalkowski / Mickler 2009), ist vor allem auf Studien zu den Arbeitsbedingungen in Branchen mit hohen Anteilen von Projektarbeit zu verweisen (vgl. Boes / Baukrowitz 2002; Eichmann 2003; Mayer-Ahuja / Wolf 2005; Marrs 2007). 
fährt. Solange die künftigen Regelungen umstritten sind, bleiben die Handlungsspielräume wesentlich erweitert: Wechselseitige Zuschreibungen von konformem versus obstruktivem Handeln werden zum taktischen Instrument in der mikropolitischen Auseinandersetzung. Die daraus resultierenden Verwicklungen können zu subjektiv empfundener Willkür und Enttäuschung führen.

Ausdruck dieser Problematik sind jene drei Integrationsfaktoren, deren Relevanz für tief greifende Veränderungsprozesse in deutschen Großunternehmen oben (Kap. 2.2) gezeigt worden ist: Mäßige Veränderungsmotivation, Desorientierung im Prozess und inkongruente Maßnahmen stehen in engem Zusammenhang mit der Unklarheit über gültige Handlungsregeln. Sie lassen unterschiedliche Ansatzpunkte zur Bewältigung des Grundproblems der (zumindest phasenweise unvermeidbaren) Simultaneität alter und neuer Normen erkennen:

- Die Kongruenz der Anpassungskomponenten trägt zur Unmissverständlichkeit der neuen Verhaltensanforderungen durch die Stimmigkeit des Maßnahmenbündels bei; sind Veränderungen breit angelegt, so besteht weniger Anlass zum Zweifel an ihrer Ernsthaftigkeit.

- Orientierung bieten eindeutige Rahmensetzungen für den Prozess des Übergangs: Je klarer und widerspruchsfreier Ziel und Plan von Veränderungsvorhaben zutage treten, umso eher können sie als Leitlinie dienen und der Gefahr machtpolitisch motivierter Auslegungen entgehen.

- Den empirischen Befunden zur Leistungsbereitschaft der Arbeitnehmer zufolge ist die Arbeitsmotivation (nicht nur beim Typus Leistungsoptimierung) generell hoch: Dieses Motivationspotenzial wird für kompetitive Kooperationsbeziehungen im Rahmen von Reorganisationsprozessen in sehr unterschiedlichem Maße erschlossen.

Mit der vorgeschlagenen Interpretation dieser Reorganisationsproblematik gerät ein grundlegender Wandel im Verhältnis von Individuum und Organisation in den Blick. Die spannungsreiche Beziehung zwischen Erneuerern und Bewahrern, die sich in Analogie zu Schumpeter als „schöpferische Zerstörung nach innen“ charakterisieren lässt, kann genauer bestimmt werden als kompetitive Kooperation: ein verdeckter Wettstreit zwischen Organisationsmitgliedern um kreative Lösungen von Organisationsproblemen. Dieser Konkurrenzmodus wird generell in dezentral organisierten Leistungsformen wirksam - etwa mit dem Anspruch der Leistungsoptimierung bei Projektarbeit. Solche unternehmerischen Impulse nach innen werden nicht marktförmig integriert, sondern im Wesentlichen - so die hier vorgeschlagene Interpretation - durch gezielte koordinative Aktivitäten der Projektarbeiter selbst.

Noch wird diese Handlungsmacht erst allmählich erkennbar und von den Projektarbeitern kaum strategisch genutzt. In dieser frühen Phase der Entwicklung ist das Bewusstsein für die neuen Handlungsmöglichkeiten wenig ausgeprägt - bei Managern wie Mitarbeitern. Offen ist deshalb nicht nur die Frage nach der Reichweite der Entwicklung, deren Schwerpunkt zurzeit im Bereich gestaltungsoffener Projektarbeit und bei zur Leistungsoptimierung bereiten Personen liegt. Ungeklärt ist vor allem, welche konkreten strategischen Optionen Manager und Mitarbeiter für die Koordination kompetitiver Leistungen wählen. Unabhängig von solchen empirisch zu bearbeitenden Forschungsfragen bleibt als theoretischer Kern der Argumentation festzuhalten: Individuen sind zu ungewöhnlich kreativen Leistungen für Organisationen in der Lage, wenn sie ihre Individualität in Abgrenzung zur Organisation über eigenständig entwickelte und kooperativ umgesetzte Vorstellungen einer produktiven Praxis definieren können. 


\section{Literatur}

Adamson, Stephen J. / Noeleen Doherty / Claire Viney (1998): The meaning of career revisited: Implications for theory and practice, in: British Journal of Management 9, S. 251-259.

Armenakis, Achilles A. / Arthur G. Bedeian (1999): Organizational change: A review of theory and research in the 1990 s, in: Journal of Management 25, S. 293-315.

Armenakis, Achilles A. / Stanley G. Harris / Kevin W. Mossholder (1993): Creating readiness for organizational change, in: Human Relations 46, S. 681-703.

Becke, Guido (2008): Soziale Erwartungsstrukturen in Unternehmen. Zur psychosozialen Dynamik von Gegenseitigkeit im Organisationswandel, Berlin.

Becker-Beck, Ulrike / Rudolf Fisch (2001): Erfolg von Projektgruppen in Organisationen - Erträge der sozialwissenschaftlichen Forschung, in: Dieter Beck / Rudolf Fisch / Birte Englich (Hrsg.), Projektgruppen in Organisationen. Praktische Erfahrungen und Erträge der Forschung, Göttingen, S. 19-42.

Bernecker, Tobias / Michael Reiß (2002): Kommunikation im Wandel. Kommunikation als Instrument des Change Managements im Urteil von Change Agents, in: zfo 71, S. 352-359.

Boes, Andreas / Andrea Baukrowitz (2002): Arbeitsbeziehungen in der IT-Industrie - ARB-IT, Berlin.

Bollinger, Heinrich (2001): Neue Formen der Arbeit - neue Formen des Gesundheitsschutzes. Das Beispiel Projektarbeit, in: WSI-Mitteilungen 54, S. 685-691.

Boltanski, Luc / Eve Chiapello (2006): Der neue Geist des Kapitalismus, Konstanz.

Buchanan, David / Richard Badham (1999): Politics and organizational change: The lived experience, in: Human Relations 52, S. 609-629.

Caldwell, Raymond (2005): Things fall apart? Discourses on angency and change in organizations, in: Human Relations 58, S. 83-114.

Capgemini (2005): Veränderungen erfolgreich gestalten - Change Management 2005. Bedeutungen, Strategien, Trends, Berlin (Broschüre), abgerufen am 17.5.2009 unter http://www.de.capgemini.com/m/de/ t1/Change_Management_2005.pdf.

Clark, Timothy / Robin Fincham (Hrsg.) (2002): Critical consulting. New perspectives on the management advice industry, Oxford.

Cohen, Michael D. / James G. March / Johan P. Olsen (1972): A garbage can model of organizational choice, in: Administrative Science Quarterly 17, S. 1-25.

Collins, David (1998): Organizational change. Sociological perspectives, London - New York.

Crant, J. Michael (2000): Proactive behavior in organizations, in: Journal of Management 26, S. 435-462.

Damanpour, Fariborz (1991): Organizational innovation: A meta-analysis of effects of determinants and moderators, in: Academy of Management Journal 34, S. 555-590.

Dawson, Patrick (2003): Understanding organizational change. The contemporary experience of people at work, Thousand Oaks, CA.

Demers, Christiane (2007): Organizational Change Theories. A Synthesis, Los Angeles u.a.

Dent, Eric B. / Susan G. Goldberg (1999): Challenging "resistance to change”, in: Journal of Applied Behavioral Science 35, S. 25-41.

Eichmann, Hubert (2003): Arbeiten in der New Economy, Wien.

Faust, Michael (2005): Managementberatung in der Organisationsgesellschaft, in: Wieland Jäger / Uwe Schimank (Hrsg.), Organisationsgesellschaft. Facetten und Perspektiven, Wiesbaden, S. 529-588.

Faust, Michael / Peter Jauch / Petra Notz (2000): Befreit und entwurzelt: Führungskräfte auf dem Weg zum ,internen Unternehmer“, München - Mering.

Fine, Gary A. (1984). Negotiated orders and organizational cultures, in: Annual Review of Sociology 10, S. 239-262.

Finstad, Nils (1998): The rhetoric of organizational change, in: Human Relations 51, S. 717-740. 
Foehrenbach, Julie / Steve Goldfarb (1990): Employee communication in the '90 s, in: Communication World 7, S. 101-106.

French, Wendell L. / Cecil H. Bell (1994): Organisationsentwicklung. Sozialwissenschaftliche Strategien zur Organisationsveränderung. 4. Auflage, Bern.

Greif, Siegfried / Bernd Runde / Ilka Seeberg (2004): Erfolge und Misserfolge beim Change Management, Göttingen.

Hall, Peter M. (1987): Interactionism and the study of social organization, in: The Sociological Quarterly 28 , S. 1-22.

Hartley, Jean / John Benington / Peter Binns (1997): Researching the role of internal change agents in the management of organizational change, in: British Journal of Management 8, S. 61-73.

Hodgson, Damian (2002): Disciplining the professional: The case of project management, in: Journal of Management Studies 39, S. 803-821.

Hodgson, Damian / Svetlana Cicmil (Hrsg.) (2006): Making projects critical, Houndmills.

Houben, Anabel / Carsten Frigge / Hans J. Pongratz / Rainer Trinczek (2007): Veränderungen erfolgreich gestalten. Repräsentative Untersuchungen über Erfolg und Misserfolg im Veränderungsmanagement, Düsseldorf (Broschüre).

Huy, Quy Nguyen (2002): Emotional balancing of organizational continuity and radical change: The contribution of middle managers, in: Administrative Science Quarterly 47, S. 31-69.

Huy, Quy Nguyen / Henry Mintzberg (2003): The rhythm of change, in: MIT Sloan Management Review 44, S. 79-84.

IBM (2007): Making change work. Erfolgsfaktoren für die Einführung von Innovation, Stuttgart (Broschüre).

Iding, Hermann (2000): Hinter den Kulissen der Organisationsberatung. Qualitative Fallstudien von Beratungsprozessen im Krankenhaus, Opladen.

Kalkowski, Peter / Otfried Mickler (2009): Antinomien des Projektmanagements. Eine Arbeitsform zwischen Direktive und Freiraum, Berlin.

Kanter, M. Rosabeth / Barry A. Stein / Todd D. Jick (1992): The challenge of organizational change. How companies experience it and leaders guide it, New York.

Kiefer, Tina (2002): Understanding the emotional experience of organizational change: Evidence from a merger, in: Advances in Developing Human Resources 4, S. 39-61.

Kiefer, Tina / Werner R. Müller / Sabine Eicken (2001): Befindlichkeit in der Chemischen Industrie, Basel: WWZ-Studie Nr. 59.

Kieser, Alfred (1996): Moden und Mythen des Organisierens, in: Die Betriebswirtschaft 56, S. 21-39.

Kieser, Alfred / Cornelia Hegele / Matthias Klimmer (1998): Kommunikation im organisatorischen Wandel, Stuttgart.

Knights, David / Hugh Willmott (Hrsg.) (2000): The Reengineering Revolution. Critical Studies of Corporate Change, London.

Kotter, John P. (1996): Leading change, Boston.

Kotthoff, Hermann (1996): Hochqualifizierte Angestellte und betriebliche Umstrukturierung. Erosion von Sozialintegration und Loyalität im Großbetrieb, in: Soziale Welt 47, S. 435-449.

Kratzer, Nick (2003): Arbeitskraft in Entgrenzung. Grenzenlose Anforderungen, erweiterte Spielräume, begrenzte Ressourcen, Berlin.

Kühl, Stefan (2002): Sisyphos im Management. Die vergebliche Suche nach der optimalen Organisationsstruktur, Weinheim.

Latniak, Erich / Anja Gerlmaier (2006): Zwischen Innovation und alltäglichem Kleinkrieg. Zur Belastungssituation von IT-Beschäftigten. IAT Report 2006-04, Gelsenkirchen. 
Leana, Carrie R. / Bruce Barry (2000): Stability and change as simultaneous experiences in organizational life, in: Academy of Management Review 25, S. 753-759.

Lewin, Kurt (1947): Frontiers in group dynamics. Concept, method and reality in social science; social equilibria and social change, in: Human Relations 1, S. 5-41.

March, James G. / Johan P. Olsen (Hrsg.) (1976): Ambiguity and choice in organisations, Bergen.

March, James G. / Robert I. Sutton (1997): Organizational performance as a dependent variable, in: Organization Science 6, S. 698-706.

Marrs, Kira (2007): Zwischen Leidenschaft und Lohnarbeit - Ein arbeitssoziologischer Blick hinter die Kulissen von Film und Fernsehen, Berlin.

Mayer-Ahuja, Nicole / Harald Wolf (Hrsg.) (2005): Entfesselte Arbeit, neue Bindungen. Grenzen der Entgrenzung in der Medien- und Kulturindustrie, Berlin.

Nicolai, Alexander / Alfred Kieser (2002): Trotz eklatanter Erfolglosigkeit: Die Erfolgsfaktorenforschung weiter auf Erfolgskurs, in: Die Betriebswirtschaft 62, S. 579-596.

Orlikowski, Wanda (1996): Improvising Organisational Transformation Over Time: A Situated Change Perspective, in: Information Systems Research 7, S. 63-92.

Ortmann, Günther / Arnold Windeler / Albrecht Becker / Hans-Joachim Schulz (1990): Computer und Macht in Organisationen. Mikropolitische Analysen, Opladen.

Ottaway, Richard N. (1983): The change agent: A taxonomy in relation to the change process, in: $\mathrm{Hu}-$ man Relations 36, S. 361-392.

Parker, Sharon K. / Nick Turner / Helen M. Williams (2006): Modeling the antecedents of proactive behavior at work, in: Journal of Applied Psychology 91, S. 636-652.

Peters, Thomas J. / Robert H. Waterman (1983): Auf der Suche nach Spitzenleistungen: Was man von den bestgeführten US-Unternehmen lernen kann, Landsberg / Lech.

Pettigrew, Andrew M. (1987): Context and action in the transformation of the firm, in: Journal of Management Studies 24, S. 649-670.

Piderit, Sandy K. (2000): Rethinking resistance and recognizing ambivalence: A multidimensional view of attitudes toward and organizational change, in: Academy of Management Review 25, S. 783-794.

Pongratz, Hans J. / Rainer Trinczek (2006): Mehr Change! Weniger Motivation? Organisatorischer Wandel im Urteil von Führungskräften und Komunikationsexperten, in: Claudia Langen / Holger Sievert (Hrsg.), Strategisch kommunizieren und führen, Gütersloh, S. 111-124.

Pongratz, Hans J. (2008): Eine Gesellschaft von Unternehmern. Expansion und Profanierung „schöpferischer Zerstörung" in kapitalistischen Ökonomien, in: Berliner Journal für Soziologie 18, S. 457-475.

Pongratz, Hans J. / G. Günter Voß (2003): Arbeitskraftunternehmer. Erwerbsorientierungen in entgrenzten Arbeitsformen, Berlin.

Pongratz, Hans J. / G. Günter Voß (Hrsg.) (2004): Typisch Arbeitskraftunternehmer? Befunde der empirischen Arbeitsforschung, Berlin.

Salaman, Graeme (2002): Understanding advice: Towards a sociology of management consultancy, in: Timothy Clark / Robin Fincham (Hrsg.), Critical consulting. New perspectives on the management advice industry, Oxford, S. 247-259.

Sauer, Dieter / Andreas Boes / Nick Kratzer (2005): Reorganisation des Unternehmens, in: SOFI, Soziologisches Forschungsinstitut u.a. (Hrsg.), Berichterstattung zur sozioökonomischen Entwicklung in Deutschland. Arbeit und Lebensweisen, Wiesbaden, S. 323-350.

Schreyögg, Georg / Christian Noss (2000): Von der Episode zum fortwährenden Prozess - Wege jenseits der Gleichgewichtslogik im Organisatorischen Wandel, in: Georg Schreyögg / Peter Conrad (Hrsg.), Organisatorischer Wandel und Transformation, Wiesbaden, S. 33-62.

Schumpeter, Joseph A. (1929): Unternehmer, in: Ludwig Elster / Adolf Weber / Friedrich Wieser (Hrsg.), Handwörterbuch der Staatswissenschaften. 8. Band. 4. gänzlich umgearbeitete Auflage, Jena, S. 476-487. 
Schumpeter, Joseph A. (1950): Kapitalismus, Sozialismus und Demokratie. 2. erweiterte Auflage, Bern.

Schumpeter, Joseph A. (1964): Theorie der wirtschaftlichen Entwicklung. Eine Untersuchung über Unternehmergewinn, Kapital, Kredit, Zins und den Konjunkturzyklus. 6. Auflage, unveränd. Nachdr. der 1934 erschienen 4. Auflage, Berlin.

Stensaker, Inger / Christine B. Meyer / Joyce Falkenberg / Anne C. Haueng (2002): Excessive change: Coping mechanisms and consequences, in: Organizational Dynamics 31, S. 296-312.

Stelling, Joan / Rue Bucher (1972). Autonomy and monitoring on hospital wards, in: The Sociological Quarterly 13, S. 431-446.

Strauss, Anselm (1978). Negotiations. Varieties, contexts, processes, and social order, San Francisco Washington - London.

Strauss, Anselm / Rue Bucher / Danuta Ehrlich / Melvin Sabshin / Leonard Schatzman (1963). The hospital and its negotiated order, in: Eliot Freidson (Hrsg.), The hospital in modern society, New York, S. 147-169.

Sullivan, Sherry E. (1999): The changing nature of careers: A review and research agenda, in: Journal of Management 25, S. 457-484.

Swedberg, Richard (1994): Markets as social structures, in: Neil Smelser / Richard Swedberg (Hrsg.), The handbook of economic sociology, Princeton, S. 255-282.

Tichy, Noel M. / Harvey A. Hornstein (1976): Stand when your number is called: An empirical attempt to classify types of social change agents, in: Human Relations 29, S. 945-967.

Witte, Eberhard / Jürgen Hauschildt / Oskar Grün (Hrsg.) (1988): Innovative Entscheidungsprozesse. Die Ergebnisse des Projekts „Columbus“, Tübingen.

Wooten, Kevin D. / Louis P. White (1989): Toward a theory of change role efficacy, in: Human Relations 42, S. 651-669.

Prof. Dr. Hans J. Pongratz

Ludwig-Maximilians-Universität München Institut für Soziologie

Konradstraße 6

80801 München

hans.pongratz@soziologie.uni-muenchen.de 